\title{
Universitários de educação física são otimistas e motivados durante a vida acadêmica?
}

Are university students of Physical Education optimistic and motivated during their academic life?

Patricia Carolina Borsato Passos

Universidade Estadual de Maringá, Brasil

borsatopassos@gmail.com

Luciane Cristina Arantes da Costa

Universidade Estadual de Maringá, Brasil

luarantes100@gmail.com

Andressa Contreira

Universidade Estadual de Maringá, Brasil

andressacontreira@gmail.com

Isabella Belem

UniCesumar, Brasil

isabellacbelem@gmail.com

Eraldo da Silva

Universidade Estadual de Maringá, Brasil

eraldoschunk@gmail.com

Lenamar Fiorese

Universidade Estadual de Maringá, Brasil

lenamarfiorese@gmail.com

\section{Resumo:}

Objetivo: Analisar a orientação de vida e a motivação acadêmica de universitários do curso de Educação Física ao longo dos quatro anos de formação inicial. Métodos: Participaram deste estudo de cunho descritivo longitudinal 24 universitários do curso de Educação Física do período integral, estudantes de uma universidade pública do sul do Brasil. Como instrumentos de pesquisa foram utilizados o Teste de orientação de vida (TOV-R), a Escala de Motivação Acadêmica e um questionário de identificação. Utilizou-se para análise estatística o teste de Friedman, o teste de U de Mann-Whitney, o Kruskal-Wallis e correlação de Spearman $(\mathrm{p}<0,05)$. Resultados: $\mathrm{O}$ nível de otimismo não se alterou ao longo dos anos, já os níveis de motivação apresentaram diferenças significativas entre os anos. Os resultados da correlação demonstraram que quanto menos horas de prática de exercício físico realizada pelos os acadêmicos, mais desmotivados eles se percebem $(\mathrm{p}=0,04)$ assim como que quanto maior a faixa etária maiores níveis de motivação intrínseca $(\mathrm{r}=0,60)$. Conclusão: Os universitários de Educação Física são otimistas durante toda a formação inicial. Ainda, constatou-se que no primeiro ano os acadêmicos são mais desmotivados quando comparados aos outros anos da formação.

Palavras-chave: Universitário, Motivação, Otimismo, Acadêmicos.

AbSTRACT:

The objective is to analyze the life orientation and the academic motivation of university students of the Physical Education course of study during the four years of initial training. Twenty-four undergraduate students of the full-time Physical Education course from a public university in southern Brazil participated in this longitudinal study. As research instruments, the Life Orientation Test (TOV-R), the Academic Motivation Scale and an identification questionnaire were used. The Friedman test, the Mann-

\section{Recepción: 11 de mayo de 2020 | Aprobación: 07 de julio de 2020 | Publicación: 01 de junio de 2020}

Cita sugerida: Passos, P. C. B., Costa, L. C. A., Contreira, A., Belem, I., da Silva, E. y Fiorese, L. (2020). Universitários de educação física são otimistas e motivados durante a vida acadêmica?. Educación Física y Ciencia, 22(2), e128. https://doi.org/10.24215/23142561e128 
Whitney U test, the Kruskal-Wallis test and the Spearman correlation $(\mathrm{p}<0.05)$ were used for the statistical analysis. The results show that the level of optimism did not change over the years, although the levels of motivation showed significant differences among the years. The results of the correlation showed that the fewer hours of physical exercise performed by the students, the more unmotivated they were $(\mathrm{p}=0.04)$ and the higher the age group, the higher the levels of intrinsic motivation $(\mathrm{r}=0.60)$. We conclude that Physical Education students are optimistic throughout initial training. However, it was verified that during the first year of training academics are more unmotivated than during other years.

KEYWORDS: University, Motivation, Optimism, Students.

\section{INTRODUÇÃ̃o}

Considerando principalmente as particularidades do contexto universitário, é necessário que sejam desenvolvidas mais pesquisas nesse âmbito. Tais particularidades englobam desde a falta de experiência face às novas demandas, tendo como consequência a adoção de hábitos não saudáveis, até o estresse psicológico, capaz de levar os jovens a uma situação de vulnerabilidade (Oliveira \& Padovani, 2014). Buscando desenvolver uma pesquisa que auxilie a saúde mental dos universitários, a psicologia positiva revelase como uma tendência a ser utilizada por estudiosos que buscam recursos psicológicos eficientes para como fatores positivos nos comportamentos desses sujeitos.

Nessa perspectiva, vem recebendo destaque na literatura a variável da psicologia positiva denominada de otimismo que, apontada como importante fator tanto na prevenção quanto nos processos de recuperação de saúde física e mental, algo que se observou em países como os EUA, a Austrália e o Reino Unido (Santos $\&$ Wechsler, 2015). No contexto acadêmico, o otimismo tem sido investigado quanto à sua relação com motivação (Shogren, Lopez, Wehmeyer, Little \& Pressgrove, 2006); Leal; Miranda \& Carmo, 2013; Costa, Nascimento Junior, Medeiros \& Vieira, 2015), comportamentos de risco (Rigoni., Costa, Passos \& Vieira, 2012), satisfação de acadêmicos (Extremera \& Rey, 2009; Larson, Good \& Fair, 2010), resiliência e bem-estar psicológico de acadêmicos de medicina (Souri \& Hasanirad, 2011), assim como o otimismo por si próprio em universitários dos EUA (Nes, Evans \& Segerstrom, 2009; Feldman \& Kubota, 2015) e Canadá (Kam \& Meyer, 2012).

Face aos estudos sobre o otimismo envolvendo os universitários encontrados, a motivação acadêmica parece ser uma variável relevante para investigação em tal contexto. Sabe-se da ocorrência de muitos estudos sobre a motivação acadêmica (Thompson \& Gaudreau, 2008; Komarraju, Karau, \& Schmeck, 2009; Koestner, Taylor, Losier \& Fichman, 2010; Clark \& Schroth, 2010; Hamdan-Mansour, Hamaideh, Arabiat \& Azzeghaiby, 2014; Réka, Kármen, Susana, Kinga, Edit \& Kinga, 2015; Costa et al., 2015; Thuy-vy \& Deci, 2016), contudo, nenhum destes analisou o otimismo e a motivação acadêmica em universitários durante toda sua formação no curso de Educação Física, caracterizando então a lacuna na qual este estudo pretende avançar.

Compreende-se que universitários de Educação Física têm suas próprias características dentro da área da saúde, dessa forma, sob a ótica do coletivo contemporâneo, não integrante de tal contexto, espera-se desse sujeito a prática regular de atividade física e hábitos saudáveis de alimentação que favoreçam a promoção da saúde. Porém, nem sempre os estudantes de Educação Física seguem as recomendações que prescreverão a seus clientes e alunos (Castro, de Souza Vale, de Aguiar \& da Silva Mattos, 2017).

Sabe-se que atividade física e alimentação são apenas alguns dos fatores que compõem a promoção da saúde. Assim, pretende-se investigar outros fatores capazes de auxiliar na saúde dos universitários de Educação Física, suas características e comportamento durante toda a formação, uma vez que outros fatores de ordens biológica, social, econômica e cultural pode influenciar a saúde (Nogueira \& Bosi, 2017), como personalidade, relacionamentos, comportamento positivo, controle do estresse e o contexto.

Previamente, deve-se esclarecer que o otimismo, considerado com uma das características da personalidade, foi investigado como resultado da orientação de vida, essa variável identifica se o sujeito tem expectativas 
mais otimistas ou pessimistas sobre eventos futuros. Nesse sentido, esta pesquisa usou o conceito de Carver \& Scheier (2001), que descrevem a percepção das pessoas sobre suas vidas (orientação de vida), sendo orientada de forma positiva (otimismo) ou negativa (pessimismo), refletindo diretamente nas expectativas que o indivíduo possui sobre eventos futuros e comportamentos praticados.

Mediante tal lacuna, o objetivo deste estudo foi analisar a orientação de vida e a motivação acadêmica de universitários do curso de Educação Física de uma universidade ao longo dos quatro anos de sua formação inicial. Com o propósito de esclarecer particularidades da população a ser estudada, foram definidos os seguintes objetivos específicos: identificar, comparar e relacionar a orientação de vida, o nível de motivação acadêmica e as características dos universitários do curso de Educação Física de uma universidade do sul do Brasil, durante todos os anos de formação.

\section{MÉTodos}

\section{Participantes do estudo}

A população alvo desta pesquisa constitui-se de alunos universitários do curso de Educação Física ingressantes no ano de 2011 e concluintes no ano de 2014 do período integral de uma universidade pública do sul do Brasil, totalizando 90 estudantes. Esta instituição de ensino superior (IES) foi selecionada de forma intencional e por conveniência (Gaya, 2016) por ser uma instituição de referência no estado do Paraná, atendendo a aproximadamente 130 municípios. Ainda, está entre as 20 universidades mais produtivas do país e conta com mais de 30 mil estudantes matriculados nos diversos cursos de graduação, sendo o curso de Educação Física o quinto maior curso em número de alunos (UEM, 2018).

O momento (ano 2011) configurava um cenário no qual o curso de Educação Física da universidade com um aspecto instigante para investigação: os acadêmicos no primeiro ano não possuíam a habilitação do curso definida, pois a partir do segundo ano é que escolheriam se continuariam o curso na habilitação de licenciatura ou bacharelado.

O primeiro ano de coleta (2011) apresentou perda amostral de 30 (33\%) alunos devido à desistência ou ao abandono do curso de Educação Física no primeiro semestre, totalizando assim 60 estudantes convidados a participar da pesquisa por meio da entrega do Termo de Consentimento Livre e Esclarecido (TCLE). Desses, foram excluídos cinco estudantes que se recusaram a responder aos protocolos, 11 que não devolveram os instrumentos respondidos no dia da coleta ou em outras tentativas de contato por meio eletrônico (e-mail e telefone) e um sujeito que não preencheu a maioria dos itens dos instrumentos. Assim, considerando tal perda amostral, em 2011 participaram do estudo 43 universitários de ambos os sexos e de diferentes faixas etárias.

No ano de 2012, nove universitários (21\%) não participaram da pesquisa por motivos de desistência do curso $(n=2)$, reprovação $(n=1)$, mudança de curso $(n=1)$, não comparecimento no dia da coleta e não retorno às tentativas de contato por meio eletrônico $(n=2)$ ou não participação por motivos pessoais $(n=3)$, totalizando 34 sujeitos. A coleta do ano de $2013(n=34)$ não apresentou perda. Já a última coleta, em 2014, teve uma perda amostral de 10 sujeitos (30\%), sendo os motivos trancamento de matrícula por doença $(n=01)$, participação no Programa Ciência Sem Fronteiras $(n=01)$, recusa $(n=07)$ e desistência $(n=1)$. Assim, o estudo foi finalizado (último ano) com 24 estudantes acompanhados ao longo de toda sua formação no curso de Educação Física (quatro anos).

\section{Instrumentos}

Para análise do otimismo, utilizou-se o Teste de orientação de vida (TOV-R) (Bekou, Tetreault, Vallerand \& Gouzet, 1999) validado para língua portuguesa por Bandeira, Bekou, Lott, Teixeira \& Rocha 
(2002), a fim de avaliar o construto do otimismo em relação a eventos futuros a partir de 10 questões, sendo três afirmações positivas (itens 1, 4 e 10), três afirmações negativas (itens 3, 7 e 9) e 4 neutras (itens 2, 5, 6 e 8), não levadas em consideração ao se analisar o instrumento. O instrumento utiliza a escala Likert de 5 pontos variando de 0 (discordo totalmente) a 4 (concordo totalmente). Para as questóes negativas, a pontuação é invertida $(0=4 ; 1=3 ; 2=2 ; 3=1 ; 4=0)$. O índice global de otimismo é calculado a partir da soma dos seis itens positivos e negativos, sendo que quanto mais alta a pontuação, maior é o grau de otimismo do indivíduo, podendo variar entre 0 e 24.

Para análise da motivação, utilizou-se a Escala de Motivação Acadêmica (Vallerand, Pelletier, Blais, Briere, Senecal \& Vallieres 1992), validada para a língua portuguesa por Guimarães \& Bzuneck (2008), composta por 29 itens e subdividida em sete dimensões: Desmotivação, Regulação externa, Regulação externa (social), Regulação introjetada, Regulação identificada, Regulação integrada e Motivação intrínseca. O estudante deve responder cada item em função de uma escala tipo Likert de 1 (nenhuma correspondência) a 7 (total correspondência).

Ainda, empregou-se um questionário de identificação (sexo, faixa etária, ano, motivo da escolha do curso, participação em projetos, estágio, prática de exercício físico e habilitação) para identificar os sujeitos e acompanhar suas atividades dentro e fora da universidade.

\section{Procedimentos}

O estudo foi autorizado pelo chefe de departamento do curso de Educação Física da referida universidade. Após a autorização departamental, o projeto foi submetido e aprovado pelo Comitê de Ética em Pesquisa Envolvendo Seres Humanos (Parecer n. 238/2011). Este estudo integrou o projeto institucional "Impacto das variáveis psicológicas sobre comportamentos relacionados à saúde”.

A coleta de dados foi conduzida pela pesquisadora responsável pelo estudo, dentro de período das aulas no ambiente acadêmico, ao longo do segundo semestre dos anos de 2011 a 2014, conforme autorização prévia da coordenação do curso bem como dos professores e assinatura do Termo de Consentimento Livre e esclarecido (TCLE) pelos acadêmicos.

\section{Análise dos dados}

O primeiro pressuposto estatístico para definir os testes mais adequados e a seleção da amostra (Marôco, 2011) para este estudo foram não aleatórios, assim, a estatística inferencial mais adequada se dá a partir da aplicação de testes não paramétricos.

Foi verificada a normalidade dos dados por meio do teste Shapiro Wilk, assim os dados foram descritos utilizando tabelas de frequência simples e apresentados através de mediana e intervalo interquartílico. Utilizou-se o teste de Friedman para comparar os níveis de motivação acadêmica em cada um dos anos de formação, sendo que o mesmo teste foi utilizado para comparar os níveis de motivação e orientação de vida entre quatro momentos $\left(1^{\circ}, 2^{\circ}, 3^{\circ}\right.$ e $4^{\circ}$ ano), durante a formação em nível superior.

Previamente às análises de comparação, os grupos foram categorizados em dois ou três grupos com base nas respostas dos questionários de identificação (com algumas perguntas abertas). Todas as respostas foram analisadas individualmente para a categorização dos dados, sem excluir nenhum dado relevante ao estudo para possibilitar as análises estatísticas. Ressalta-se que para fins de categorização, foram analisadas as semelhanças e diferenças entre todas as variáveis. A faixa etária estabelecida para este estudo especificamente teve como referencial teórico o estudo de validação da Escala de Motivação Acadêmica, cujos autores utilizaram as seguintes faixas de idade: até 19 anos, entre 20 e 25 anos, entre 26 e 30 anos e acima de 31 anos 
de idade (Guimarães \& Bzuneck, 2008). Entretanto, este estudo não precisou unir as duas últimas categorias devido ao baixo número de sujeitos, passando a ser nomeado "Acima de 25 anos de idade".

Para a comparação de dois grupos foi utilizado o teste de U-Mann-Whitney considerando as seguintes variáveis: sexo (feminino ou masculino), realização de estágio na área (sim ou não), prática de atividade física (sim e não), tipo de prática (exercício físico ou esporte), motivo da escolha do curso no primeiro ano (pessoal e outro), habilitação (bacharelado ou licenciatura) e participação em projetos (sim ou não). Já para comparar três grupos aplicou-se o teste de Kruskal-Wallis considerando a comparação múltipla das distribuições de médias de todas as ordens, a partir das seguintes variáveis: faixa etária (até 19 anos, 20 e 25 anos e acima de 25 anos de idade), tempo de prática semanal de exercício físico ( 1 a 3 horas, 4 a 6 horas e 7 ou mais horas) e o nível de motivação e a orientação de vida. Ainda, quando sujeitos apareciam em somente duas dessas categorias, utilizou-se o teste de U de Mann-Whitney.

Para verificar a relação entre as variáveis dependentes foi utilizado o coeficiente de correlação de Spearman, classificando em correlação moderada $(0,40$ a 0,59$)$ e correlação forte (acima de 0,60$)$ (Mitra \& Lankford, 1999). As análises foram realizadas usando software SPSS versão 20.0 adotando a significância de $\mathrm{p}<0,05$.

\section{Resultados}

A Tabela 1 contêm os dados descritivos das características dos estudantes de Educação Física por meio de frequência e percentual. No primeiro ano somente um acadêmico realizava estágio como seu trabalho, mas a regulamentação do estágio de estudantes de Educação Física dessa universidade não permite a realização de estágio remunerado. Outro dado relevante é o fato de que todos os participantes desta pesquisa estavam inseridos em algum projeto (pesquisa, ensino ou extensão) quando estavam no segundo ano do curso.

TABELA 1

Descrição das variáveis em frequência e percentual dos universitários de Educação Física do $1^{\circ}$ ano ao $4^{\circ}$ ano, em função das variáveis categóricas.

\begin{tabular}{|c|c|c|c|c|}
\hline Variáveis & $\begin{array}{l}1^{\circ} \text { ano } \\
\mathrm{n}=24 \\
f(\%)\end{array}$ & $\begin{array}{l}2^{\circ} \text { ano } \\
\mathrm{n}=24 \\
f(\%)\end{array}$ & $\begin{array}{l}3^{\circ} \\
\text { ano } \\
\mathrm{n}=24 \\
f(\%)\end{array}$ & $\begin{array}{l}4^{\circ} \\
\text { ano } \\
\mathrm{n}=24 \\
f(\%)\end{array}$ \\
\hline $\begin{array}{l}\text { Faixa etária Até } 19 \\
\text { anos } 20 \text { a } 25 \text { anos } \\
\text { Acima de } 25 \text { anos }\end{array}$ & $\begin{array}{l}12 \\
(50) 11 \\
(46) 01 \\
(04)\end{array}$ & $\begin{array}{l}05 \\
(21) 18 \\
(75) 01 \\
(04)\end{array}$ & $\begin{array}{l}04 \\
(17) \\
16 \\
(66) \\
04 \\
(17)\end{array}$ & $\begin{array}{l}-20 \\
(83) \\
04 \\
(17)\end{array}$ \\
\hline $\begin{array}{l}\text { Sexo Masculino } \\
\text { Feminino }\end{array}$ & $\begin{array}{l}10 \\
(42) 14 \\
(58)\end{array}$ & $\begin{array}{l}10 \\
(42) 14 \\
(58)\end{array}$ & $\begin{array}{l}10 \\
(42) \\
14 \\
(58)\end{array}$ & $\begin{array}{l}10 \\
(42) \\
14 \\
(58)\end{array}$ \\
\hline $\begin{array}{l}\text { Habilitação } \\
\text { Licenciatura } \\
\text { Bacharelado }\end{array}$ & -- & $\begin{array}{l}13 \\
(54) 11 \\
(46)\end{array}$ & $\begin{array}{l}12 \\
(50) \\
12 \\
(50)\end{array}$ & $\begin{array}{l}10 \\
(42) \\
14 \\
(58)\end{array}$ \\
\hline $\begin{array}{l}\text { Motivo da escolha do } \\
\text { curso Pessoal Outro } \\
\text { motivo }\end{array}$ & $\begin{array}{l}08 \\
\text { (33) } 16 \\
(67)\end{array}$ & $\begin{array}{c}23 \\
(96) 01 \\
(04)\end{array}$ & $\begin{array}{l}23 \\
(96) \\
01 \\
(04)\end{array}$ & $\begin{array}{l}23 \\
(96) \\
01 \\
(04)\end{array}$ \\
\hline $\begin{array}{l}\text { Trabalho em } \\
\text { estágio/projeto Sim } \\
\text { Não }\end{array}$ & $\begin{array}{l}01 \\
(04) 23 \\
(96)\end{array}$ & $\begin{array}{l}09 \\
(37) 15 \\
(63)\end{array}$ & $\begin{array}{l}10 \\
(42) \\
14 \\
(58)\end{array}$ & $\begin{array}{l}15 \\
(63) \\
09 \\
(37)\end{array}$ \\
\hline $\begin{array}{l}\text { Participação em } \\
\text { projetos Sim Não }\end{array}$ & $\begin{array}{l}01 \\
(04) 23 \\
(96)\end{array}$ & $\begin{array}{c}24 \\
(100) \\
00(00)\end{array}$ & \begin{tabular}{l}
\multicolumn{1}{c}{23} \\
$(96)$ \\
01 \\
$(04)$
\end{tabular} & $\begin{array}{l}19 \\
(79) \\
05 \\
(21)\end{array}$ \\
\hline $\begin{array}{l}\text { Realiza exercícios } \\
\text { físicos Sim Nắo }\end{array}$ & $\begin{array}{l}23 \\
(96) 01 \\
(04)\end{array}$ & $\begin{array}{l}19 \\
(79) 05 \\
(21)\end{array}$ & $\begin{array}{l}19 \\
(79) \\
05 \\
(21)\end{array}$ & $\begin{array}{l}18 \\
(75) \\
06 \\
(25)\end{array}$ \\
\hline $\begin{array}{l}\text { Tipo de prática } \\
\text { Exercício Físico Esporte }\end{array}$ & $\begin{array}{l}12 \\
(50) 08 \\
(33)\end{array}$ & $\begin{array}{l}09 \\
(38) 10 \\
(42)\end{array}$ & $\begin{array}{l}11 \\
(46) \\
08 \\
(33)\end{array}$ & $\begin{array}{l}10 \\
(42) \\
08 \\
(33)\end{array}$ \\
\hline $\begin{array}{l}\text { Tempo de prática } \\
\text { semanal } 1 \text { a } 3 \text { horas } 4 \text { a } \\
6 \text { horas } 7 \text { horas ou mais }\end{array}$ & $\begin{array}{l}05 \\
(21) 10 \\
(42) 04 \\
(17)\end{array}$ & $\begin{array}{l}05 \\
(21) 04 \\
(17) 10 \\
(42)\end{array}$ & $\begin{array}{l}02 \\
(08) \\
08 \\
(33) \\
09 \\
(37)\end{array}$ & $\begin{array}{l}02 \\
08) \\
07 \\
(29) \\
09 \\
(38)\end{array}$ \\
\hline
\end{tabular}

Nota: Em dois grupos (tipo de prática e tempo de prática) houve participantes que não responderam esses itens da coleta de dados do questionário de identificação. Assim, alguns grupos tiveram números menores que o $n$ total de sujeitos (24 sujeitos). 
Nota-se que os dados da habilitação Tabela 1 não constam no primeiro ano uma vez que o ingresso dos acadêmicos no vestibular do ano de início desta pesquisa (2011) não tinha a definição da habilitação, ou seja, os acadêmicos só decidiam ao final do primeiro ano, optando pela habilitação em licenciatura ou bacharelado.

A Tabela 2 apresenta a comparação entre motivação e orientação de vida durante os quatro anos de formação dos universitários de Educação Física. $\mathrm{O}$ nível de otimismo não se alterou significativamente ao longo dos anos, indicando que os universitários mantiveram-se mais otimistas durante os quatro anos de formação, considerando a escala do índice de otimismo ( 0 a 24) com medianas entre 17 e 18 em todos os momentos. Os níveis de motivação apresentaram diferenças significativas entre os anos $(\mathrm{p}=0,01)$, indicando que os acadêmicos do $1^{\circ}$ ano demonstraram a desmotivação maior $(\mathrm{Md}=4)$ e menor na motivação regulação integrada $(\mathrm{Md}=3)$ em relação aos demais anos.

TABELA 2

Comparação das variáveis (motivação e orientação de vida) dos universitários de Educação Física, de uma universidade do Paraná, em função dos quatro anos de curso $(n=24)$.

\begin{tabular}{|c|c|c|c|c|}
\hline Variáveis & $\begin{array}{l}1^{\circ} \text { ano Md } \\
(\mathrm{Q} 1-\mathrm{Q} 3)\end{array}$ & $\begin{array}{l}2^{\circ} \text { ano Md } \\
(\mathrm{Q} 1-\mathrm{Q} 3)\end{array}$ & $\begin{array}{l}3^{\circ} \text { ano } \mathrm{Md} \\
(\mathrm{Q} 1-\mathrm{Q} 3)\end{array}$ & $\begin{array}{l}4^{\circ} \text { ano } \mathrm{Md} \\
(\mathrm{Q} 1-\mathrm{Q} 3)\end{array}$ \\
\hline Desmotiv ação & $4(2-5)^{a}$ & $\begin{array}{l}1(1-1) \\
\mathrm{a} / \mathrm{b} / \mathrm{c} / \mathrm{d} / \mathrm{e}\end{array}$ & $\begin{array}{l}1(1-1) \\
a / b / c / d / e\end{array}$ & $\begin{array}{l}1(1-2) \\
\text { a/b/c/d/e }\end{array}$ \\
\hline $\begin{array}{l}\text { Regulação } \\
\text { Extrínseca }\end{array}$ & $5(4-6)^{b / d}$ & $3(2-4)^{a / j}$ & $4(2-5)^{c / g}$ & $4(2-5)^{d / h}$ \\
\hline Externa Social & $\begin{array}{l}2(1-3) \\
a / b / c\end{array}$ & $\begin{array}{l}1(1-2) \\
\mathrm{f} / \mathrm{g} / \mathrm{h} / \mathrm{i}\end{array}$ & $\begin{array}{l}1(1-3) \\
\mathrm{f} / \mathrm{g} / \mathrm{h} / \mathrm{i}\end{array}$ & $\begin{array}{l}2(1-2) \\
\mathrm{f} / \mathrm{g} / \mathrm{h} / \mathrm{i}\end{array}$ \\
\hline $\begin{array}{l}\text { Regulação } \\
\text { Introjetada }\end{array}$ & $2(1-4)^{f}$ & $4(2-5)^{b / f / k}$ & $3(2-4)^{a / j}$ & $3(2-5)^{a}$ \\
\hline $\begin{array}{l}\text { Regulação } \\
\text { Identificada }\end{array}$ & $3(1-4)^{d / e}$ & $4(3-5)^{d / h /}$ & $4(3-5)^{d / h}$ & $4(2-5)^{b / f}$ \\
\hline $\begin{array}{l}\text { Regulação } \\
\text { Integrada }\end{array}$ & $3(2-4)$ & $\begin{array}{l}6(5-6) \\
e / i / j / k\end{array}$ & $5(4-6)^{e / 1 / j}$ & $5(3-6)^{e / i}$ \\
\hline $\begin{array}{l}\text { Motivação } \\
\text { Intrínseca }\end{array}$ & $5(4-6)^{c / e / f}$ & $5(3-6)^{c / g}$ & $4(3-5)^{b / f}$ & $4(3-5)^{c / g}$ \\
\hline $\begin{array}{l}\text { Orientação de } \\
\text { Vida (OV) }\end{array}$ & $17(13-21)$ & $17(14-20)$ & $18(15-20)$ & $18(14-20)$ \\
\hline
\end{tabular}

Nota: Teste de Friedman = comparação dos quatro momentos descritos no texto; as comparações das regulações dos níveis de motivação dentro de cada ano do curso são apresentadas as diferenças estatisticamente significativas com as letras A, B, C, D, E, F, G, H, I, J e K.

Quando comparados os níveis de motivação para identificar o perfil motivacional dos acadêmicos no $1^{\circ}$ ano (Tabela 2), os resultados demonstraram um nível de regulação social significativamente menor $(\mathrm{Md}=$ $2,0)$, enquanto a motivação intrínseca foi a maior $(\mathrm{Md}=5,0)$ em relação às demais regulações.

Verificou-se que os acadêmicos do $2^{\circ}$ ano demonstraram menores níveis de motivação na regulação social $(\mathrm{Md}=1,0)$ e a desmotivação $(\mathrm{Md}=1,0)$, enquanto a maior mediana foi a da regulação integrada $(\mathrm{Md}=6,0)$. Na comparação dos níveis de motivação dos acadêmicos do $3^{\circ}$ ano (Tabela 2), verificou-se que as regulações se mantiveram iguais, as menores foram a regulação social $(\mathrm{Md}=1,0)$ e a desmotivação $(\mathrm{Md}=1,0)$, já a maior foi a regulação integrada $(\mathrm{Md}=5,0)$. No $4^{\circ}$ ano evidenciou-se que as menores medianas dos níveis de motivação continuaram entre a regulação social $(\mathrm{Md}=2,0)$ e a desmotivação $(\mathrm{Md}=1,0)$.

A Tabela 3 apresenta as comparações da motivação em função dos motivos de escolha do curso e tempo de prática semanal de exercício físico dos universitários do $1^{\circ}$ ano do curso de Educação Física. Os resultados demonstraram que o nível de desmotivação foi maior quando a escolha do curso não se deu por motivo pessoal $(\mathrm{Md}=4,3)$ assim como apresentou níveis de regulação integrada $(\mathrm{Md}=5,4)$ e motivação intrínseca $(\mathrm{Md}=5,7)$ maior quando o motivo foi pessoal $(\mathrm{Md}=5,4)$. 


\section{TABELA 3}

Comparação dos níveis de motivação dos universitários do primeiro ano do curso de Educação Física ( $\mathrm{n}=24)$ em função dos motivos de escolha do curso e horas de prática de exercício físico.

\begin{tabular}{|c|c|c|c|c|c|c|c|}
\hline $\begin{array}{l}\text { Variáveis } \\
\text { Grupos }\end{array}$ & $\begin{array}{l}\text { DES } \\
\text { Md }\end{array}$ & $\begin{array}{l}\mathrm{RE} \\
\mathrm{Md}\end{array}$ & $\begin{array}{l}\text { SOC } \\
\text { Md }\end{array}$ & $\begin{array}{l}\text { RIN } \\
\text { Md }\end{array}$ & $\begin{array}{l}\text { IDEN } \\
\text { Md }\end{array}$ & $\begin{array}{l}\text { RINT } \\
\text { Md }\end{array}$ & $\begin{array}{l}\text { MINT } \\
\text { Md }\end{array}$ \\
\hline \multicolumn{8}{|l|}{$\begin{array}{l}\text { Motivo da } \\
\text { escolha }\end{array}$} \\
\hline Pessoal & 1,0 & 3,4 & 1,7 & 3,3 & 4,5 & 5,4 & 5,7 \\
\hline Outro & 4,3 & 4,6 & 2,7 & 3,1 & 3,0 & 3,2 & 4,5 \\
\hline$P$ & $0,04^{*}$ & 0,81 & 0,15 & 0,35 & 0,26 & $0,01^{*}$ & $0,03^{*}$ \\
\hline $\begin{array}{l}\text { Prática } \\
\text { semanal } \\
1 \text { a } 3 \\
\text { horas }\end{array}$ & $4,0^{\mathrm{a}}$ & 4,2 & 3,0 & 2,8 & 3,0 & 2,5 & 6,0 \\
\hline $\begin{array}{l}4 \text { a } 6 \\
\text { horas }\end{array}$ & $4,5^{b}$ & 4,6 & 2,9 & 3,4 & 3,5 & 3,5 & 4,3 \\
\hline $\begin{array}{l}7 \mathrm{~h} \text { ou } \\
\text { mais }\end{array}$ & $1,6^{\mathrm{a} / \mathrm{b}}$ & 3,3 & 1,9 & 3,1 & 4,0 & 4,8 & 5,3 \\
\hline$P$ & $0,02^{\star}$ & 0,28 & 0,34 & 0,10 & 0,57 & 0,06 & 0,23 \\
\hline
\end{tabular}

\footnotetext{
${ }^{*} \mathrm{p}<0,05$ (Teste U de Man Whitney e Kruskal-Wallis). Nota: DES=desmotivação,

$\mathrm{RE}=$ regulação extrínseca, $\mathrm{SOC}=$ regulação externa social, $\mathrm{RIN}=$ regulação introjetada, IDEN=regulação identificada, RINT=regulação integrada e MINT=motivação intrínseca.
}

Houve diferença estatisticamente significativa (Tabela 3) quando comparadas as horas de prática de exercício físico, demonstrando que quanto mais horas de prática menor é o nível de desmotivação $(\mathrm{p}=0,02)$.

A Tabela 4 apresenta nas comparaçóes em três grupos os acadêmicos do $2^{\circ}$ ano com diferenças significativas. Os resultados revelaram que os acadêmicos ingressantes em estágio ou trabalho na área são mais motivados por regulação extrínseca $(\mathrm{Md}=4,2)$ e regulação identificada $(\mathrm{Md}=6,0)$ em relação aos que não realizam ações na área. Na comparação entre os que realizam e os que não realizam prática de exercício físico, os acadêmicos apresentam maiores níveis de motivação intrínseca $(\mathrm{Md}=5,0)$ quando praticam exercício físico $(\mathrm{p}=0,02)$.

$\mathrm{Na}$ comparação entre os grupos de horas de prática de exercício físico (Tabela 4), os acadêmicos indicam maiores níveis de regulação extrínseca $(\mathrm{Md}=4,3)$ e regulação identificada $(\mathrm{Md}=5,5)$ no grupo que se dedica a 7 horas semanais ou mais à prática de exercício físico. 
TABELA 4

Comparação dos níveis de motivação dos universitários do segundo ano

do curso de Educação Física ( $\mathrm{n}=24)$ em função de realizar estágio ou trabalho na área, realizar exercício físico e horas de prática de exercício físico.

\begin{tabular}{|c|c|c|c|c|c|c|c|}
\hline $\begin{array}{l}\text { Variáveis } \\
\text { Grupos }\end{array}$ & $\begin{array}{l}\text { DES } \\
\text { Md }\end{array}$ & $\begin{array}{l}\mathrm{RE} \\
\mathrm{Md}\end{array}$ & $\begin{array}{l}\text { SOC } \\
\text { Md }\end{array}$ & $\begin{array}{l}\text { RIN } \\
\text { Md }\end{array}$ & $\begin{array}{l}\text { IDEN } \\
\text { Md }\end{array}$ & $\begin{array}{l}\text { RINT } \\
\text { Md }\end{array}$ & $\begin{array}{l}\text { MINT } \\
\text { Md }\end{array}$ \\
\hline \multicolumn{8}{|l|}{ Estágio/Trabalho } \\
\hline $\operatorname{Sim}$ & 1,3 & 4,2 & 2,0 & 4,0 & 6,0 & 6,3 & 5,7 \\
\hline Não & 1,2 & 2,8 & 1,7 & 3,5 & 4,0 & 6,0 & 4,3 \\
\hline$P$ & 0,41 & $0,01^{*}$ & 0,29 & 0,12 & $0,02^{*}$ & 0,19 & 0,08 \\
\hline \multicolumn{8}{|l|}{$\begin{array}{l}\text { Realizar } \\
\text { exercício físico }\end{array}$} \\
\hline $\operatorname{Sim}$ & 1,2 & 3,2 & 2,0 & 4,0 & 4,5 & 6,3 & 5,0 \\
\hline Nẫo & 1,8 & 2,2 & 1,7 & 3,0 & 5,0 & 6,3 & 2,3 \\
\hline$P$ & 0,18 & 0,36 & 0,73 & 0,73 & 0,10 & 0,10 & $0,02^{*}$ \\
\hline $\begin{array}{l}\text { Prática semanal } \\
1 \text { a } 3 \text { horas }\end{array}$ & 1,3 & $3,2^{\mathrm{a}}$ & 1,7 & 3,2 & $4,0^{\mathrm{a}}$ & 6,0 & 4,0 \\
\hline 4 a 6 horas & 1,1 & $2,3^{\mathrm{b}}$ & 1,2 & 4,2 & $3,3^{b}$ & 6,1 & 5,2 \\
\hline 7 horas ou mais & 1,3 & $4,3^{a / b}$ & 2,0 & 4,2 & $5,5^{\mathrm{a} / \mathrm{b}}$ & 6,3 & 5,5 \\
\hline$p$ & 0,90 & $0,04^{*}$ & 0,18 & 0,21 & $0,03^{*}$ & 0,88 & 0,25 \\
\hline
\end{tabular}

${ }^{*} \mathrm{p}<0,05$ (Teste U de Mann Whitney e Kruskal-Wallis). Nota: DES=desmotivação, $\mathrm{RE}=$ regulação extrínseca, $\mathrm{SOC}=$ regulação externa social, $\mathrm{RIN}=$ regulação introjetada, IDEN=regulação identificada, RINT=regulação integrada e MINT=motivação intrínseca.

Os resultados da Tabela 5 evidenciaram que os acadêmicos do terceiro ano praticantes de exercício físico obtiveram maiores níveis de motivação extrínseca social $(\mathrm{Md}=2,3)$ e regulação identificada $(\mathrm{Md}=4,5)$ assim como maior mediana no índice de orientação de vida em relação àqueles que não realizam exercício físico. Quando comparados os grupos de acadêmicos praticantes de esporte e os praticantes de exercício físico, os níveis de motivação de regulação integrada foram maiores para os que praticam esporte $(\mathrm{Md}=6,3)$.

Ainda (Tabela 5), os acadêmicos praticantes de exercício físico ou esporte por 4 a 6 horas semanais apresentaram maior índice de orientação de vida em relação aos que realizam 7 horas ou mais de prática. Os resultados identificaram que os acadêmicos do $3^{\circ}$ ano de Educação Física com habilitação em bacharelado possuem maiores níveis de motivação de regulação introjetada $(\mathrm{Md}=3,9)$ e regulação identificada $(\mathrm{Md}=5,0)$ em comparação aos acadêmicos da licenciatura. 
TABELA 5

Comparação dos níveis de motivação e orientação de vida (TOV) dos universitários do terceiro ano do curso de Educação Física $(n=24)$ em função da habilitação, realizar exercício físico, tipo de prática e horas de prática de exercício físico.

\begin{tabular}{|c|c|c|c|c|c|c|c|c|}
\hline Variáveis & DES & RE & $\mathrm{SOC}$ & RIN & IDEN & RINT & MINT & TOV \\
\hline Grupos & Md & Md & & & & & & \\
\hline \multicolumn{9}{|l|}{ Habilitação } \\
\hline Bacharelado & 1,5 & 4,7 & 2,0 & 3,9 & 5,0 & 5,5 & 4,5 & 3,3 \\
\hline$p$ & 0,55 & 0,06 & 0,41 & $0,01^{*}$ & $0,01^{*}$ & 0,12 & 0,26 & 0,84 \\
\hline \multicolumn{9}{|c|}{$\begin{array}{l}\text { Realizar exercício } \\
\text { físico }\end{array}$} \\
\hline $\operatorname{Sim}$ & 1,5 & 3,8 & 2,3 & 3,3 & 4,5 & 5,0 & 4,3 & 3,5 \\
\hline Nã̃o & 1,2 & 3,2 & 1,0 & 3,2 & 2,5 & 4,3 & 2,7 & 2,0 \\
\hline$p$ & 0,29 & 0,29 & $0,01^{*}$ & 0,08 & $0,03^{*}$ & 0,18 & 0,10 & $0,03^{*}$ \\
\hline \multicolumn{9}{|l|}{$\begin{array}{l}\text { Tipo de } \\
\text { prática }\end{array}$} \\
\hline $\begin{array}{l}\text { Exercício } \\
\text { físico }\end{array}$ & 1,3 & 4,4 & 2,3 & 3,3 & 4,0 & 5,0 & 4,3 & 3,5 \\
\hline Esporte & 1,6 & 3,1 & 2,4 & 3,6 & 4,5 & 6,3 & 4,3 & 3,5 \\
\hline$p$ & 0,54 & 0,31 & 0,77 & 0,71 & 0,84 & $0,03^{*}$ & 0,31 & 0,90 \\
\hline \multicolumn{9}{|l|}{$\begin{array}{l}\text { Prática } \\
\text { semanal }\end{array}$} \\
\hline 1 a 3 horas & 1,5 & 3,1 & 2,7 & 3,4 & 3,3 & 5,8 & 4,5 & - \\
\hline 4 a 6 horas & 1,9 & 3,2 & 2,7 & 3,5 & 4,3 & 5,3 & 4,2 & 4,0 \\
\hline $\begin{array}{l}7 \text { ou mais } \\
\text { horas }\end{array}$ & 1,3 & 4,4 & 2,0 & 3,3 & 5,0 & 5,0 & 4,3 & 3,0 \\
\hline$p$ & 0,17 & 0,45 & 0,40 & 0,94 & 0,18 & 0,70 & 0,94 & $0,03^{*}$ \\
\hline
\end{tabular}

${ }^{*} \mathrm{p}<0,05$ (Teste U de Mann Whitney e Kruskal-Wallis). Nota: DES=desmotivação, RE=regulação extrínseca, $S O C=$ regulação externa social, RIN=regulação introjetada, IDEN=regulação

identificada, RINT=regulação integrada; MINT=motivação intrínseca, e TOV= orientação de vida.

Verifica-se (Tabela 6) que os acadêmicos do $4^{\circ}$ ano apresentaram diferenças estatisticamente significativas na comparação de cinco variáveis. Os universitários do sexo masculino têm maiores níveis de motivação de regulação integrada $(\mathrm{Md}=5,8)$ e intrínseca $(\mathrm{Md}=5,0)$ em comparação ao sexo feminino. $\mathrm{Na}$ comparação entre as habilitações, notou-se que os níveis de motivação tiveram alterações aproximadas da autodeterminação uma vez que os resultados dos acadêmicos de bacharelado têm maiores níveis de motivação de regulação identificada $(\mathrm{Md}=5,3)$ e integrada $(\mathrm{Md}=5,8)$ considerando os acadêmicos de licenciatura.

Os resultados (Tabela 6) referentes à comparação entre os acadêmicos praticantes de exercício físico e os não praticantes demonstraram maiores níveis de motivação por regulação integrada $(\mathrm{Md}=5,7)$ e motivação intrínseca $(\mathrm{Md}=4,5)$ para os que realizam exercício físico. $\mathrm{Na}$ comparação entre faixa etária, os acadêmicos inseridos acima de 25 anos se percebem mais motivados intrinsecamente $(\mathrm{Md}=5,0)$ em relação aos de 20 a 25 anos de idade $(\mathrm{Md}=3,7)$. Os resultados evidenciaram que quanto menos horas de prática de exercício físico os acadêmicos realizam mais desmotivados eles se percebem $(\mathrm{p}=0,04)$. 
TABELA 6

Comparação dos níveis de motivação dos universitários do quarto ano do curso de Educação Física (n=24) em função do sexo, habilitação, realizar exercício físico, faixa etária e horas de prática de exercício físico.

\begin{tabular}{|c|c|c|c|c|c|c|c|}
\hline $\begin{array}{l}\text { Variáveis } \\
\text { Grupos } \\
\end{array}$ & $\begin{array}{l}\text { DES } \\
\mathbf{M d}\end{array}$ & $\begin{array}{l}\mathrm{RE} \\
\mathrm{Md}\end{array}$ & $\begin{array}{l}\text { SOC } \\
\text { Md }\end{array}$ & $\begin{array}{l}\text { RIN } \\
\text { Md }\end{array}$ & $\begin{array}{l}\text { IDEN } \\
\text { Md }\end{array}$ & $\begin{array}{l}\text { RINT } \\
\text { Md }\end{array}$ & $\begin{array}{l}\text { MINT } \\
\text { Md }\end{array}$ \\
\hline \multicolumn{8}{|l|}{ Sexo } \\
\hline Masculino & 1,8 & 3,7 & 2,2 & 2,9 & 4,5 & 5,8 & 5,0 \\
\hline Feminino & 1,9 & 4,7 & 2,2 & 2,2 & 3,8 & 4,3 & 3,7 \\
\hline$p$ & 0,62 & 0,47 & 0,93 & 0,28 & 0,70 & $0,03^{*}$ & $0,01^{*}$ \\
\hline \multicolumn{8}{|l|}{ Habilitação } \\
\hline Licenciatura & 2,3 & 4,2 & 2,0 & 2,7 & 2,8 & 3,6 & 3,7 \\
\hline Bacharelado & 1,6 & 4,3 & 2,9 & 4,6 & 5,3 & 5,8 & 4,5 \\
\hline$p$ & 0,08 & 0,66 & 0,15 & 0,07 & $0,01^{*}$ & $0,02^{*}$ & 0,17 \\
\hline \multicolumn{8}{|l|}{$\begin{array}{l}\text { Realizar } \\
\text { exercício } \\
\text { físico }\end{array}$} \\
\hline $\operatorname{Sim}$ & 1,8 & 4,2 & 2,3 & 4,1 & 4,3 & 5,7 & 4,5 \\
\hline Não & 2,1 & 3,9 & 1,7 & 2,7 & 3,5 & 3,6 & 3,2 \\
\hline$p$ & 0,62 & 0,25 & 0,17 & 0,05 & 0,07 & $0,01^{*}$ & $0,04^{*}$ \\
\hline \multicolumn{8}{|l|}{ Faixa etária } \\
\hline Até 19 anos & - & - & - & - & - & - & - \\
\hline $\begin{array}{l}20 \text { a } 25 \\
\text { anos }\end{array}$ & 1,9 & 4,2 & 2,0 & 3,1 & 4,0 & 5,5 & 3,7 \\
\hline $\begin{array}{l}\text { Acima de } \\
25 \text { anos }\end{array}$ & 1,7 & 3,9 & 3,0 & 4,1 & 4,8 & 6,3 & 5,0 \\
\hline$p$ & 0,79 & 0,97 & 0,06 & 0,73 & 0,43 & 0,15 & $0,01^{*}$ \\
\hline \multicolumn{8}{|l|}{$\begin{array}{l}\text { Prática } \\
\text { semanal }\end{array}$} \\
\hline 1 a 3 horas & $3,4^{a / b}$ & 4,6 & 2,5 & 4,0 & 2,3 & 5,2 & 5,2 \\
\hline 4 a 6 horas & $2,0^{b / c}$ & 5,0 & 2,0 & 4,2 & 4,5 & 5,5 & 4,7 \\
\hline $\begin{array}{l}7 \text { ou mais } \\
\text { horas }\end{array}$ & $1,3^{\mathrm{a} / \mathrm{c}}$ & 3,4 & 2,3 & 4,0 & 5,0 & 5,8 & 4,3 \\
\hline$p$ & $0,04^{*}$ & 0,56 & 0,88 & 0,76 & 0,23 & 0,30 & 0,15 \\
\hline
\end{tabular}

${ }^{*} \mathrm{p}<0,05$ (Teste U de Mann Whitney e Kruskal-Wallis). Nota: DES=desmotivação, $\mathrm{RE}=$ regulação extrínseca, $\mathrm{SOC}=$ regulação externa social, $\mathrm{RIN}=$ regulação introjetada, IDEN=regulação identificada, RINT=regulação integrada e MINT=motivação intrínseca.

A Tabela 7 apresenta as correlações entre motivação com orientação de vida, faixa etária e horas praticadas de exercício físico por semana em todos os anos dos acadêmicos de Educação Física avaliados. Os acadêmicos do $1^{\circ}$ ano apresentaram correlação moderada e significativa $(\mathrm{p}=0,03)$ demonstrando que quanto mais horas de prática de exercício físico maiores são os níveis de motivação de regulação integrada $(\mathrm{r}=0,48)$. Os acadêmicos do $2^{\circ}$ ano também demonstraram correlação moderada e significativa $(p=0,02)$ evidenciando que quanto mais horas de prática de exercício físico maiores foram os níveis de motivação por regulação identificada $(\mathrm{r}=0,52)$. Ainda, verificou-se que quando os acadêmicos se encontravam no $2^{\circ}$ ano, quanto maior a faixa etária maiores eram os níveis de motivação intrínseca $(r=0,60)$, estabelecendo uma correlação forte e significativa $(\mathrm{p}=0,02)$.

Os resultados dos acadêmicos do $3^{\circ}$ ano (Tabela 7 ) indicaram correlações moderadas e significativas entre a faixa etária e os níveis de motivação de regulação extrínseca $(\mathrm{r}=0,44)$ e regulação introjetada $(\mathrm{r}=0,41)$, demonstrando que quanto maior a faixa etária, maiores são os níveis destas regulações. Já quando os acadêmicos se encontravam no último ano do curso (Tabela 7$)$, houve correlação significativa ( $\mathrm{p}=0,01$ ) (semelhante ao $2^{\circ}$ ano) moderada positiva considerando a faixa etária $(\mathrm{r}=0,50)$, demonstrando assim que 
os acadêmicos se percebem com níveis maiores de motivação intrínseca quando integram o grupo de faixa etária maior.

TABELA 7

Coeficiente de correlação dos níveis de motivação com orientação de vida (TOV), faixa etária (F.E.) e horas de exercício físico (H.E.F.) dos universitários de Educação

Física de uma universidade no sul do Brasil, durante todos os anos de formação.

\begin{tabular}{|c|c|c|c|c|c|c|c|c|c|c|c|c|}
\hline Momentos & & $\begin{array}{l}1^{\circ} \\
\text { ano }\end{array}$ & & & $\begin{array}{l}2^{\mathbf{O}} \\
\text { ano } \\
\end{array}$ & & & $\begin{array}{l}3^{\circ} \\
\text { ano } \\
\end{array}$ & & & $\begin{array}{l}4^{\circ} \\
\text { ano } \\
\end{array}$ & \\
\hline Variáveis & TOV & F.E. & H.E.F. & TOV & F.E. & H.E.F. & TOV & F.E. & H.E.F. & TOV & F.E. & H.E.F. \\
\hline Desmotivação & 0,02 & 0,20 & $-0,42$ & $-0,11$ & $-0,06$ & $-0,01$ & $-0,03$ & 0,05 & $-0,29$ & $-0,11$ & $-0,06$ & $-0,58^{*}$ \\
\hline $\begin{array}{l}\text { Regulação } \\
\text { Extrínseca }\end{array}$ & $-0,03$ & $-0,02$ & $-0,24$ & 0,29 & 0,17 & 0,39 & $-0,18$ & $0,44^{*}$ & 0,28 & 0,12 & $-0,02$ & $-0,25$ \\
\hline Externa Social & $-0,35$ & 0,16 & $-0,28$ & 0,08 & 0,04 & 0,35 & 0,08 & 0,22 & $-0,31$ & 0,08 & 0,39 & $-0,11$ \\
\hline $\begin{array}{l}\text { Regulação } \\
\text { Introjetada }\end{array}$ & $-0,01$ & 0,19 & 0,17 & 0,12 & 0,12 & 0,40 & $-0,04$ & $0,41^{*}$ & 0,05 & 0,17 & 0,08 & $-0,10$ \\
\hline $\begin{array}{l}\text { Regulação } \\
\text { Identificada }\end{array}$ & 0,09 & $-0,03$ & 0,24 & 0,28 & 0,07 & $0,52^{*}$ & 0,13 & 0,24 & 0,40 & 0,18 & 0,18 & 0,29 \\
\hline $\begin{array}{l}\text { Regulação } \\
\text { Integrada }\end{array}$ & 0,06 & 0,02 & $0,48^{*}$ & 0,09 & 0,23 & 0,11 & $-0,20$ & 0,02 & $-0,18$ & $-0,11$ & 0,31 & 0,28 \\
\hline $\begin{array}{l}\text { Motivação } \\
\text { Intrínseca }\end{array}$ & 0,12 & $-0,26$ & $-0,24$ & 0,16 & $0,60^{*}$ & 0,38 & $-0,05$ & 0,15 & $-0,02$ & $-0,04$ & $0,50^{*}$ & $-0,45$ \\
\hline
\end{tabular}

* Spearman

\section{Discussão}

O otimismo é uma variável relacionada às expectativas que o indivíduo possui sobre eventos futuros e comportamento praticados (Carver \& Scheier, 2001). Os resultados (Tabela 2) dos índices de otimismo identificados pelo teste de orientação de vida não apresentaram diferença ao longo do tempo, corroborando com a literatura, que aponta o otimismo como um traço de personalidade construído a partir de experiências na infncia (Weinberg \& Gould, 2016).

Thompson \& Gaudreau (2008) realizaram uma pesquisa com delineamento longitudinal a partir da qual afirmaram que a característica otimista ou pessimista parece ser difícil de alterar, dados estes que vêm ao encontro dos evidenciados nesta pesquisa (Tabela 2). Nes, Evans \& Segerstrom (2009) empregaram o mesmo delineamento, reforçando a credibilidade dos achados deste estudo, e concluíram que o otimismo disposicional é geralmente considerado um traço da personalidade raramente passível de modificação.

Ainda, pode-se positivamente verificar que os universitários se perceberam mais otimistas ao longo de todos os anos de formação em Educação Física (Tabela 2). Esse achado pode ser compreendido como um aspecto positivo considerando o apontamento de Aspinwall \& Taylor (1992), que estabelecem a orientação de vida mais otimista como fator que leva os estudantes a se ajustem mais facilmente ao ambiente universitário.

Quando analisados os níveis de motivação ao longo dos anos de formação (Tabela 2), os acadêmicos do $1^{\circ}$ ano obtiveram um nível de desmotivação maior que nos outros anos do curso e menores níveis de motivação por regulação integrada, sendo esta a forma mais autônoma de motivação extrínseca (Ryan \& Deci, 2008). Isso demonstrando que esses universitários não estavam realizando atividades em coerência com outros aspectos de si mesmo, comportamento que leva o indivíduo a ser menos autodeterminado. Tais dados chamam a atenção de estudiosos para os acadêmicos do $1^{\circ}$ ano, que demonstraram estar mais longe da motivação autodeterminada em relação a outros momentos da formação. 
Existe produção de conhecimento indicando como buscar a motivação autodeterminada com descrição em estudos baseados na Teoria da Autodeterminação (TAD). Resultados positivos devido à atuação dos professores na promoção da autonomia de estudantes universitários proporcionaram uma regulação mais internalizada (Reeve, 2014) e maior regulação autônoma para a aprendizagem. Além de aumentar a percepção de competência e diminuir a ansiedade, os alunos também obtiveram melhores notas ao final do curso (Black \& Deci, 2004).

Em tratando-se de identificar o nível de motivação dos acadêmicos em cada ano de formação, verificou-se que para os acadêmicos, considerando os anos em uma análise transversal (Tabela 2), os níveis mais baixos de regulação extrínseca social e de desmotivação nos últimos três anos $\left(2^{\circ}, 3^{\circ}\right.$ e $\left.4^{\circ}\right)$ contrariam os resultados da pesquisa realizada com estudantes do curso de Educação Física, cujos resultados revelaram acadêmicos do terceiro ano com maiores níveis de desmotivação que os do primeiro, segundo e quarto anos (Spittle, Jackson \& Casey, 2009).

Os resultados contrários podem ser explicados pelas diferenças culturais entre universitários de diferentes países, conforme apontando no estudo cross-cultural de Shin \& Kelly (2013), os quais evidenciaram que a relação com a família modera a influência da motivação intrínseca entre universitários dos EUA, já os universitários da Coréia do Sul apresentaram relação direta do otimismo com a identidade vocacional.

Ao considerar os sete níveis de motivação avaliados dentro de cada ano como se fossem sujeitos de grupos independentes (não considerar o delineamento longitudinal para este resultado), os acadêmicos do $1^{\circ}$ ano demonstraram maiores níveis de motivação intrínseca, enquanto os acadêmicos do $2^{\circ}$ ano e $3^{\circ}$ ano tiveram maiores níveis de motivação por regulação integrada (Tabela 2). Destaca-se a importância do delineamento longitudinal para verificar a motivação ao longo do tempo considerando o caráter contraditório dos resultados do estudo por consequência da análise realizada. Costa et al. (2015) também concluíram que os alunos do primeiro ano do curso Educação Física são mais motivados intrinsecamente. Tais achados contrastam com os resultados da comparação dos níveis de motivação dos universitários ao longo dos anos, os quais apontaram maior motivação no primeiro ano em relação aos demais anos.

Quando comparados os níveis de motivação em função do motivo de escolha do curso verificou-se que os acadêmicos do $1^{\circ}$ ano (Tabela 3 ) cuja escolha do curso se deu por motivo pessoal estavam mais motivados por regulação integrada, demonstrando que o ingresso na universidade estava de acordo com seus objetivos e valores, comportamento este que leva o indivíduo a ser mais autodeterminado (Ryan \& Deci, 2008). Já os acadêmicos que escolheram o curso por outro motivo apresentaram maiores níveis de desmotivação. Tais resultados corroboram com uma meta-análise que avaliou o processo de escolha com a motivação de alunos, concluindo que a motivação intrínseca pode ser melhorada quando os sujeitos escolhem aquilo que desejam para sua vida (Patall, Cooper \& Robinson, 2008).

Os acadêmicos do $2^{\circ}$ ano (Tabela 4) que estavam realizando estágio ou trabalhando na área demonstraram maiores níveis de motivação por regulação extrínseca e identificada. Destaca-se que a regulação externa é a forma mais controlada da motivação (a pessoa age para obter ou evitar consequências externas), todavia, a motivação identificada ocorre em situações nas quais o indivíduo participa da atividade pelo reconhecimento e pela valorização (Ryan \& Deci, 2004). Esses achados demonstram a importância de se realizar estágio ou trabalho na área para alcançar a motivação dos universitários durante o segundo ano do curso.

Os acadêmicos do $3^{\circ}$ ano (Tabela 5) apresentaram maiores níveis de motivação acadêmica regulada de forma integrada (motivação menos regulada de forma extrínseca) para o grupo que praticava alguma modalidade esportiva. Esse é um achado que revela que além dos inúmeros benefícios proporcionados pela prática do esporte, para estes universitários foi também um fator positivo na motivação acadêmica. No sentido de reforçar a relevância da pratica de esporte, um estudo conduzido por Jones \& Parker (2013) demonstrou que experiências positivas na prática esportiva de jovens do Reino Unido explicam um percentual da resistência mental desses jovens. 
Somente os acadêmicos do $3^{\circ}$ ano (Tabela 5) apresentaram maiores índices de otimismo, evidenciando que quando realizavam exercício físico e se dedicavam à prática de exercício ou esporte por 4 a 6 horas semanais, estes se perceberam mais otimistas sobre suas vidas. Um achado positivo a respeito de uma percepção mais otimista diz respeito a tais universitários de Educação Física também se percebem mais saudáveis (Rigoni et al., 2012). Isso demonstra a importância da percepção mais otimista que pode favorecer comportamentos positivos dos universitários, como a prática regular de exercício físico ou esporte.

No $4^{\circ}$ ano (Tabela 6) os acadêmicos do sexo masculino apresentaram níveis maiores de motivações integrada e intrínseca, demonstrando que estes universitários frequentam o curso de Educação Física por perceberem os benefícios pessoais, sentirem prazer e gosto nas atividades desenvolvidas (Ryan \& Deci, 2000). Um estudo de Yukseloglu \& Karaguven (2013) realizado na Turquia envolvendo estudantes do ensino médio, com idade entre 17 a 22 anos, evidenciou que os estudantes do sexo masculino apresentaram níveis mais altos de motivação que o sexo feminino.

Outra particularidade entre os acadêmicos do $4^{\circ}$ ano (Tabela 6) foi a faixa etária acima de 25 anos de idade, os quais são mais motivados intrinsecamente. Esses resultados podem ser explicados ao partir do estudo de Gonçalves (2014) envolvendo universitários portugueses com idade superior aos 23 anos. Seus achados apontam que o maior tempo de experiência de vida faz com que os alunos mais velhos possuam recursos internos e externos capazes de facilitar a adaptação frente a situações novas, exigidas no contexto acadêmico.

A habilitação foi uma variável interveniente para os acadêmicos do $4^{\circ}$ ano (Tabela 6) e para os acadêmicos do $3^{\circ}$ ano (Tabela 5), demonstrando que a habilitação de bacharelado teve maiores níveis de motivação acadêmica para os universitários durante esses anos. Isso evidencia que a motivação desses estudantes teve regulações mais externas (introjetada), o que pode explicar os comportamentos universitários para evitar a culpa ou a busca por reconhecimento social (Ryan \& Deci, 2008). Porém, ao longo do tempo essas regulações foram mais internas (identificada e integrada), pois os resultados dos universitários no $4^{\circ}$ ano do bacharelado (Tabela 6). Os resultados a respeito da habilitação podem ser comparados aos do estudo de Costa et al. (2015) que identificaram os acadêmicos do curso de Educação Física com habilitação em bacharelado com motivação por regulação externa (social) maior em relação aos de licenciatura.

Ao verificar o grupo dos praticantes de exercício físico (Tabelas 4, 5 e 6) tal prática parece ser relevante para o acadêmico de Educação Física, a partir do $2^{\circ}$ ano do curso até o último ano ( $4^{\circ}$ ano). $\mathrm{O}$ fator mostrou-se um aspecto positivo para motivação acadêmica, conforme os resultados de cada um desses anos, mesmo que as regulações não foram sempre intrínsecas, em todos os momentos. Sobre as diferenças entre as regulações durante os anos, é importante compreender que diferentes aspectos podem auxiliar ou prejudicar a motivação no ambiente acadêmico, fazendo com que os alunos não consigam manter uma regulação mais interna de causalidade percebida, conforme a TAD.

Contudo, os resultados anteriores demonstram uma fragilidade nos níveis de motivação dos acadêmicos no $3^{\circ}$ ano, dados estes que merecem ser mais profundamente investigados. Evidências já apontadas pelos pesquisadores australianos Spittle, Jackson \& Casey (2009) indicaram que os acadêmicos da Educação Física do terceiro ano apresentaram níveis maiores de desmotivação que os do primeiro, segundo e quarto anos.

Os resultados a respeito das horas de prática (Tabela 4 e 6) não recebe atenção simplesmente por reforçar a afirmação consensual da literatura sobre atividade física, ou seja, os inúmeros benefícios da prática de exercício físico. Os achados avançam à medida que mostram que quanto maior a quantidade de horas dedicadas ao exercício físico ou esporte, maior a motivação acadêmica para estudantes de Educação Física. Assim, as horas de prática de exercício podem ser um dos fatores externos que influenciam de forma significativa a motivação acadêmica, já que a regulação externa indica que a percepção de controle motivacional está fora do indivíduo (Lens, Matos \& Vansteenkiste, 2008).

Quando correlacionados os níveis de motivação com a orientação de vida, faixa etária e horas de exercício físico por semana (Tabela 7) revelou-se uma relação positiva forte e moderada entre faixa etária e níveis de motivação acadêmica. No $2^{\circ}$ ano e $3^{\circ}$ ano os acadêmicos demonstraram que quanto maior a faixa etária 
maiores são os níveis de motivação acadêmica (motivação intrínseca, regulação extrínseca e introjetada). Esses resultados podem ser compreendidos como um fator positivo para o contexto acadêmico conforme o estudo de Koestner et al. (2010) cujos resultados identificaram que os acadêmicos que endossaram as regulações identificada, externa e introjetada, como um resultado positivo encontraram razões para prosseguir em uma educação em nível de graduação.

Destaca-se que a orientação de vida não se correlacionou com nenhuma das variáveis neste estudo. Porém, os resultados de Hamdan-Mansour et al. (2014) contrastam com nossos achados, uma vez que investigaram universitários da Jordânia e encontraram associação entre motivação acadêmica intrínseca, suporte familiar, satisfação de vida e otimismo. Assim, percebe-se que a orientação de vida necessita ser mais profundamente investigada em universitários, bem como é necessário também colher outros dados desses sujeitos (nível socioeconômico) em relação às suas atividades dentro (horas de estudo, desempenho e satisfação) e fora da universidade (horas de trabalho, remuneração e metas) utilizando o delineamento longitudinal. Outros estudos já apontaram para a necessidade de estudos longitudinais envolvendo um período mais longo de acompanhamento (Thompson \& Gaudreau, 2008; Nes, Evans \& Segerstrom, 2009; Koestner et al., 2010). Ainda, sugere-se que futuros estudos busquem um número maior de sujeitos para generalização dos resultados, mesmo que pertences a mesma ou diferentes universidades, sendo esse o principal aspecto que limitou esta pesquisa.

\section{CONCLUSÃo}

Conclui-se os universitários se percebem mais otimistas durante toda a formação em Educação Física e apresentam no primeiro ano maior desmotivação e menor regulação integrada em relação aos outros anos, reforçando assim que estudiosos da área devem direcionar seus esforços a esses sujeitos para promoverem a motivação acadêmica mais regulada internamente ao longo dos anos de formação.

Quando analisados os níveis de motivação acadêmica somente dos estudantes no primeiro ano sem considerar o delineamento longitudinal, a motivação aparece pelo gosto e prazer em suas atividades no contexto acadêmico. Já para $\mathrm{p} 2^{\circ}$ e $3^{\circ}$ anos do curso, a motivação acadêmica se deu por realizarem atividades em coerência com outros aspectos de si mesmos, como seus objetivo e valores. E em todos os anos a regulação social foi mais baixa que as outras dimensões, demonstrando que esses universitários não são motivados para obter recompensa ou reconhecimento social, achados especificamente resultantes das análises dentro de cada ano.

Na comparação de grupos, os achados considerando somente cada um dos anos revelaram que o estudante que opta por um curso por um motivo pessoal percebe-se mais motivado no primeiro ano do curso. Se no segundo ano realiza estágio na área, este parece ser um fator que influencia nos níveis de regulação, mesmo sendo regulação extrínseca. Para o terceiro ano a prática de modalidade esportiva parece auxiliar a motivação mais autônoma e no quarto e último ano do curso os acadêmicos do sexo masculino e da faixa etária de acima de 25 anos de idade são mais motivados de forma autodeterminada, realizando a atividade acadêmica por prazer e por acreditar que estar de acordo com seus objetivos e valores.

Houve semelhança no $3^{\circ}$ ano e $4^{\circ}$ ano quando considerada a habilitação em bacharelado, sendo terceiro ano mais regulado extrinsecamente, passando para uma motivação mais autodeterminada no último ano. Para os acadêmicos dos últimos três anos a prática de exercício físico pareceu motivar o estudante, fazendo com que as regulações extrínsecas modificassem na direção da motivação intrínseca entre os anos.

Observou-se ainda que os acadêmicos em três anos diferentes apresentam motivação acadêmica maior quando praticavam mais horas de exercício físico ou esporte. Ao analisar a relação entre as variáveis, nota-se que os acadêmicos do $2^{\circ}$ ano e $3^{\circ}$ ano demonstram que quanto maior a faixa etária maiores são os níveis de motivação acadêmica, desde a motivação por gosto e prazer pela atividade e posteriormente até para evitarem a culpa e pressões externas. 
Diante do exposto é notável a importância dos achados para intervenções com essa população, promover a autonomia dos universitários, prática regular de exercício físico ou esporte, estimulando o relacionamento social, ressaltar a importância das diferentes habilitações no curso de Educação Física para o desenvolvimento da sociedade e competência profissional nos diferentes campos de trabalho, no intuito de promover regulações mais autodeterminadas da motivação acadêmica.

\section{REFERÊNCIAS BIBLIOGRÁFICAS}

Aspinwall, L. G., \& Taylor, S. E. (1992). Modeling cognitive adaptation: a longitudinal investigation of the impact of individual differences and coping on college adjustment and performance. Journal of personality and social psychology, 63(6), 989.

Bandeira, M., Bekou, V., Lott, K. S., Teixeira, M. A., \& Rocha, S. S. (2002). Validação transcultural do Teste de Orientação da Vida (TOV-R). Estudos de Psicologia, 7(2), 251-258.

Bekou, V., Tetreault, B., Vallerand, R., \& Gouzet, F. (1999). Developpement et validation d'une version canadiennefrancaise du "Life Orientation Test".

Carver, C. S., \& Scheier, M. F. (2001). On the self-regulation of behavior. Cambridge University Press.

Castro, J. B. P., de Souza Vale, R. G., de Aguiar, R. S., \& da Silva Mattos, R. (2017). Perfil do estilo de vida de universitários de Educação Física da cidade do Rio de Janeiro. Revista Brasileira de Ciência e Movimento, 25(2), 73-83.

Clark, M. H., \& Schroth, C. A. (2010). Examining relationships between academic motivation and personality among college students. Learning and individual differences, 20(1), 19-24.

Costa, L. A., do Nascimento Junior, J. R. A., Medeiros, A. I. A., \& Vieira, L. F. (2015). Estudo dos fatores motivacionais e orientação de vida de universitários. Cinergis, 16(2).

Deci, E. L., \& Ryan, R. M. (2008). Facilitating optimal motivation and psychological well-being across life's domains. Canadian Psychology/Psychologie canadienne, 49(1), 14.

Deci, E. L., \& Ryan, R. M. (Eds.). (2004). Handbook of self-determination research. University Rochester Press.

Extremera, N., Durán, A., \& Rey, L. (2009). The moderating effect of trait meta-mood and perceived stress on life satisfaction. Personality and Individual Differences, 47(2), 116-121.

Feldman, D. B., \& Kubota, M. (2015). Hope, self-efficacy, optimism, and academic achievement: Distinguishing constructs and levels of specificity in predicting college grade-point average. Learning and Individual Differences, $37,210-216$.

Gaya, A. (2016). Projetos de pesquisa cientifica e pedagógica: o desafio da iniciação cientifica. Belo Horizonte: Casa da educação física.

Gonçalves, P. F. D. (2014). Transição e adaptação dos novos públicos ao Ensino Superior: o caso dos maiores de 23 na Universidade do Minho. (Tese de Doutorado em Ciências da Educação). Instituto de Educação, Braga, Portugal.

Guimarães, S. É. R. (2008). Propriedades psicométricas de um instrumento para avaliação da motivação de universitários. Ciências \& Cognição, 13(1).

Hamdan-Mansour, A. M., Hamaideh, S. H., Arabiat, D. H., \& Azzeghaiby, S. N. (2014). Psychosocial correlates of motivation for academic accomplishment among university students. Procedia-Social and Behavioral Sciences, 159, 32-36.

Jones, M. I., \& Parker, J. K. (2013). What is the size of the relationship between global mental toughness and youth experiences?. Personality and Individual Differences, 54(4), 519-523.

Kam, C., \& Meyer, J. P. (2012). Do optimism and pessimism have different relationships with personality dimensions? A re-examination. Personality and Individual Differences, 52(2), 123-127.

Koestner, R., Taylor, G., Losier, G. F., \& Fichman, L. (2010). Self-regulation and adaptation during and after college: A one-year prospective study. Personality and Individual Differences, 49(8), 869-873. 
Komarraju, M., Karau, S. J., \& Schmeck, R. R. (2009). Role of the Big Five personality traits in predicting college students' academic motivation and achievement. Learning and individual differences, 19(1), 47-52.

Larson, M. J., Good, D. A., \& Fair, J. E. (2010). The relationship between performance monitoring, satisfaction with life, and positive personality traits. Biological Psychology, 83(3), 222-228.

Leal, E. A., Miranda, G. J., \& Carmo, C. R. S. (2013). Teoria da autodeterminação: uma análise da motivação dos estudantes do curso de ciências contábeis. Revista Contabilidade \& Finanças, 24(62), 162-173.

Lens, W., Matos, L., \& Vansteenkiste, M. (2008). Professores como fontes de motivação dos alunos: o quê e o porquê da aprendizagem do aluno. Educação, 31(1), 17-20.

Marôco, J. (2011). Análise estatística: com utilização do SPSS. 5. ed. Lisboa: ReportNumber.

Mitra, A. (1999). Research methods in park, recreation, and leisure services. Champaign: Sagamore Publishing.

Nogueira, J. A. D., \& Bosi, M. L. M. (2017). Saúde Coletiva e Educação Física: distanciamentos e interfaces. Ciência \& Saúde Coletiva, 22, 1913-1922.

Oliveira, N. R. C. D., \& Padovani, R. D. C. (2014). Saúde do estudante universitário: uma questão para reflexão. Ciência \& Saúde Coletiva, 19, 995-996.

Patall, E. A., Cooper, H., \& Robinson, J. C. (2008). The effects of choice on intrinsic motivation and related outcomes: a meta-analysis of research findings. Psychological bulletin, 134(2), 270.

Reeve, J. (2014). Understanding motivation and emotion. John Wiley \& Sons.

Réka, J., Kármen, D., Susana, F., Kinga, K. J., Edit, M., \& Kinga, S. (2015). Implications of motivational factors regarding the academic success of full-time and distance learning undergraduate students: A Self-Determination Theory perspective. Procedia-Social and Behavioral Sciences, 187, 50-55.

Rigoni, P. A. G., da Costa, L. C. A., Belem, I. C., Passos, P. C. B., \& Vieira, L. F. (2012). Orientação de vida e comportamentos de risco para a saúde em universitários: uma análise sob o olhar da psicologia positiva. Journal of Physical Education, 23(3), 361-368.

Santos, M. C., \& Wechsler, S. M. (2015). Análise das publicações científicas sobre otimismo em saúde no último triênio. Psicologia Argumento, 33(83).

Shin, Y. J., \& Kelly, K. R. (2013). Cross - cultural comparison of the effects of optimism, intrinsic motivation, and family relations on vocational identity. The Career Development Quarterly, 61(2), 141-160.

Shogren, K. A., Lopez, S. J., Wehmeyer, M. L., Little, T. D., \& Pressgrove, C. L. (2006). The role of positive psychology constructs in predicting life satisfaction in adolescents with and without cognitive disabilities: An exploratory study. The Journal of Positive Psychology, 1(1), 37-52.

Solberg Nes, L., Evans, D. R., \& Segerstrom, S. C. (2009). Optimism and College Retention: Mediation by Motivation, Performance, and Adjustment 1.Journal of Applied Social Psychology, 39(8), 1887-1912.

Souri, H., \& Hasanirad, T. (2011). Relationship between resilience, optimism and psychological well-being in students of medicine. Procedia-Social and Behavioral Sciences, 30, 1541-1544.

Spittle, M., Jackson, K., \& Casey, M. (2009). Applying self-determination theory to understand the motivation for becoming a physical education teacher. Teaching and Teacher Education, 25(1), 190-197.

Thompson, A., \& Gaudreau, P. (2008). From optimism and pessimism to coping: The mediating role of academic motivation. International Journal of Stress Management, 15(3), 269.

Thuy-vy, T. N., \& Deci, E. L. (2016). Can it be good to set the bar high? The role of motivational regulation in moderating the link from high standards to academic well-being.Learning and Individual Differences, 45, 245-251.

Universidade Estadual de Maringá. Assessoria de Planejamento. Coordenadoria de Planos e Informações. Base de dados 2018: ano base 2017. Maringá-PR: UEM-ASP, 2018.

Vallerand, R. J., Pelletier, L. G., Blais, M. R., Briere, N. M., Senecal, C., \& Vallieres, E. F. (1992). The Academic Motivation Scale: A measure of intrinsic, extrinsic, and amotivation in education. Educational and psychological measurement, 52(4), 1003-1017. 
Weinberg, R. S., \& Gould, D. (2016). Fundamentos da psicologia do esporte e do exercício. Artmed editora. 6a ed. Porto Alegre: Artmed.

Yukseloglu, S. M., \& Karaguven, M. H. (2013). Academic motivation levels of technical high school students. ProcediaSocial and Behavioral Sciences, 106, 282-288. 